\title{
Visual search time based on stochastic serial and parallel processings
}

TAKEHIRO UENO

OSAKA CITY UNIVERSITY, JAPAN

Two stochastic latency models were developed in order to account for random fluctuations of the search time that the $S$ consumed when he was asked to search for a single target number or one of two (or three) target numbers through the sets of small two-digit numbers randomly distributed on a white square. It was shown that the search time distribution for a single target number can be adequately described in terms of McGill's two-step serial processing model. The parallel processing model whose structure is exponential was found to fit approximately the search time distributions for the first target number that the $S$ detects among the two (or three) target numbers predesignated. This model, however, does not satisfactorily handle the long search times.

Interest has recently been awakened in the study designed to determine whether a human subject processes sensory messages in serial or parallel modes. The properties which are related to the modes of information processing have been examined by utilizing data concerning reaction time (Egeth, 1966; Estes \& Wessel, 1966; Neisser, 1963a; Sternberg, 1967), backward masking (Weisstein, 1966), choice behavior (Fried et al, 1965), and so on. Some of the accumulated data strongly suggest that the mode of information processing by a human subject is not a unique one, but rather he can use both serial and parallel processing modes, depending upon the conditions employed. Therefore, an adequate approach to the problem of serial vs parallel processings must be made in the form of examining under what condition either of the two modes holds.

The purpose of the present investigation is twofold. The first is to demonstrate how much effect the difference in the simple searching task required of the subject has on distributions of the search time observed, and the second is to analyze the obtained data in terms of the stochastic models which are based on the modes of serial and parallel processings.

\section{Apparatus}

\section{METHOO}

The $\mathbf{S}$ sat in front of a table and observed binocularly a stimulus exposure field of a TKK tachistoscope through its aperture. The exposure field, the brightness of which was approximately 300 lux, was a square $30 \mathrm{~cm}$ on a side, and the viewing distance from the S's eye to its center position was $70 \mathrm{~cm}$.

A microswitch was used to calibrate search time, and it was placed on the table in front of the S. The onset and offset of the microswitch were made by the S's right finger tip. The onset of the microswitch synchronized with both the exposure of a stimulus display in the tachistoscope and the start of a timer by which search time could be measured; the offset of the microswitch brought about both the disappearance of the display and the stop of the timer. This system made it possible to equate the exposure time of the display with S's search time, which was to be measured. The timer permitted recordings of time in units of $0.01 \mathrm{sec}$.

\section{Stimulus Material}

The stimulus materials were 98 two-digit numbers which ranged from 01 through 10 to 98 . These numerical stimuli were located in a square $(27 \mathrm{x}$ $27 \mathrm{~cm})$ drawn with black India ink on a white card, and they were typed by means ' of a Lion automatic numbering machine (Model $\mathrm{C}$ ) in the following manner: The square was divided into. 81 hypothetical cells each of which was a possible small square, $3 \mathrm{~cm}$ on a side; the 98 two-digit numbers were arranged in such a way that on the average 1.2 twodigit numbers might be distributed over each cell. Mathematically speaking, the spatial distribution of the two-digit numbers followed a Poisson distribution $e^{-1.2}(1.2)^{x} / x$ !, where $x$ represents the number of the two-digit numbers that falls in a cell. In the present experiment, the maximum value of $x$ was four.

A typed two-digit number was $0.7 \mathrm{~cm}$ wide and $0.5 \mathrm{~cm}$ high; therefore, it subtended $0.57^{\circ}$ and $0.41^{\circ}$ in visual angle, respectively, when it was presented at the exposure field of the tachistoscope. The color of the typed numbers was black. A complete set of stimuli consisted of 20 cards; each card differed from other cards with respect to the spatial location of a particular two-digit number in a square.

\section{Procedure}

At the outset, the $S$ was given general information concerning the present experiment as follows: (1) The $S$ 's task was to search for a single target number or one of two (or three) target numbers through the set of two-digit numbers randomiy distributed on a white display. (2) Since the timing of the search began with the pressing of the microswitch and ended with its release, it was necessary for the $S$ to lift his finger from the microswitch as soon as 
he detected the target number. (3) No restriction was imposed on the starting point nor on the direction of the S's search.

The following three searches were required for each of the Ss: (1) One target search-The E designated a single two-digit number as a target from the 98 two-digit numbers, and then the $\mathrm{S}$ was asked to look for the target number as quickly as possible until he discovered it on a white square. (2) Two target search-Two of the 98 two-digit numbers were selected as target numbers. The $S$ was instructed to continue to search for the two numbers designated until he detected either of them. In this search, it was well emphasized that if $S$ found one of the two targets, he did not have to look for the other. (3) Three target search-Three target numbers were chosen from among the 98 two-digit numbers. As in two target search, the $S$ was told to discover rapidly any one of the three targets.

Samples of target numbers were randomly selected under the restriction that each $\mathrm{S}$ never look for the same target number twice in the series of searches. Furthermore, in each search two sets of target numbers were prepared for each $S$ in order to cancel his preference for numerical characteristics (for example, 30 and 67 in one target search).

In each of the three searches, the $E$ always verbally informed the $S$ of the target number for which he was to search at the beginning of each trial, and, after a few seconds, a search began. In the case of three target search, the $S$ was instructed to memorize carefully the numbers designated. The $\mathrm{S}$ knew in advance that his search was being timed.

Two preliminary trials were run on each search for each $S$ using target numbers other than those in the main experiment. Following these trials, $S$ made 20 searches, i.e., one search per stimulus card. The first 10 trials were concerned with one set of target numbers and the second with the other set. If $\mathrm{S}$ could not find a target after $1 \mathrm{~min}$ of search had elapsed, then no time was recorded for that trial. The card was presented again later. The experimental order of three searches was random for each S. There were $5 \mathrm{~min}$ rest breaks between the three searches. All sessions were carried out in a dimly lit room.

\section{Subjects}

Twenty undergraduate students, majoring in psychology at Osaka City University, served as Ss. All had normal or corrected normal vision.

\section{Data Processing}

\section{RESULTS}

Since the time that the $S$ consumes to search for a particular target or one of two (or three) targets, i.e., latency, can be regarded as stochastic by nature, we hereafter treat it as a continuous random vari- able, T. From theoretical as well as data processing points of view (McGill, 1963; Parzen, 1962, p. 168), it is convenient to use the function

$$
\log [1-F(t)]=\log \left[\int_{t}^{\infty} f(x) d x\right]
$$

where $F(t)$ represents the distribution function of $T$ and $f(x)$ represents its probability density function.

Data obtained are illustrated in Fig. 1, where the ordinate denotes Equation (1), i.e., the logarithm of the area in the tail of a distribution beyond an arbitrary time t. Each distribution consists of 400 search times, since 20 Ss made one search for each of 20 stimulus cards. In Fig. 1, the three curves are based on predictions from the models developed below.

Inspection of Fig. 1 reveals that there is a striking contrast between the search for one target and for one of two or three targets. In one target search, the early stage (under 9 sec approximately) shows systematic departures from linearity, whereas linearity is realized at the later stage. Search time distributions for two and three target search, on the other hand, indicate that they turn out to be linear at the early stage, whereas at the stage beyond approximately $9 \mathrm{sec}$ they show nonlinearity. Based upon these features, it is quite reasonable to infer that the stochastic mechanism which identifies the underlying process of one target search is one thing; the mechanism for two or three target search is another. In fact, the stochastic models based on two different modes of information processing seem to fit these data, as will be seen later.

\section{Models}

We shall first of all sketch a conceptual model for search behaviors. Imagine the $S$ as a searchlight-like device. In the search for one target number, the $\mathrm{S}$ is assumed to behave in much the same way as a single searchlight moving over a dark display while a detector continuously examines the illuminated details, and, as soon as the searchlight

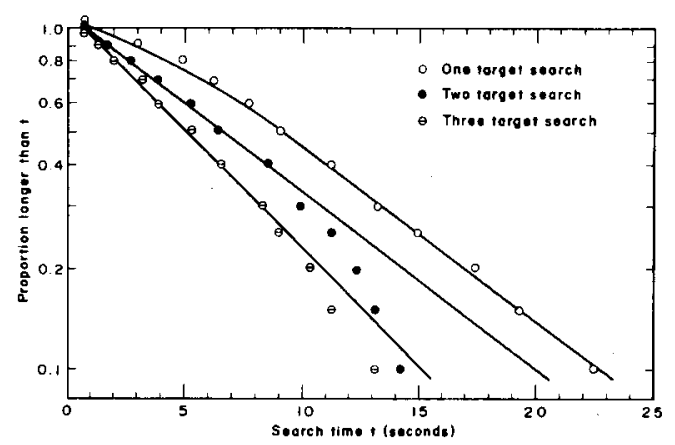

Fig. 1. Visual search time distributions for small two-digit numbers located randomly among 98 alternatives on a $27 \mathrm{~cm}$ white square. Each of the data is based on 400 search times of $20 \mathrm{Ss}$. 
hits the target, some form of decision mechanism operates. It seems very plausible to suppose that a single searchlight implies a searching process or channel which is associated with the single target number the $S$ wants to detect. A serial processing model would be the simplest of the models that represent such a single process.

In the case of two target search, on the other hand, the $S$ is assumed to have two independent searchlights. A search, then, is supposed to terminate when either of them catches one of the predesignated targets. We may here regard two searchlights as two searching processes. Furthermore, it is quite natural to assume that these processes are carried out in parallel until one of the two targets is found. This reasoning leads to the development of a parallel processing model with two channels for two target search. The same is assumed to hold for three target search.

Serial Processing Model. Data of one target search could be analyzed in terms of the serial processing model. The mathematical structure of the model is identical to the two-step serial processing model formulated by McGill (1963, pp. 324-327) concerning a target search situation which is very similar to the present one. Following McGill, suppose that the observed search time $t$ consists of the sum of two independent components, $t_{1}$ and $t_{2}$, called reaction time (short time) and decision time (long time), respectively. Then we have

$$
t=t_{1}+t_{2}
$$

Note that there is a fixed dead time for each of the three searches shown in Fig. 1. To account for this time, the two components $t_{1}$ and $t_{2}$ are assumed to have sufficiently small dead times, ${ }^{T} 1$ and $T_{2}$, respectively. For the sake of simplicity, we now assume that $\tau_{1}$ and $\tau_{2}$ are nonrandom variables.

If the probability density functions of $t_{1}$ and $t_{2}$ are given by

$$
f\left(t_{1}\right)= \begin{cases}\beta e^{-\beta\left(t_{1}-\tau_{1}\right)}, & t_{1} \geq \tau_{1} \\ 0 & , t_{1}<\tau_{1}\end{cases}
$$

and

$$
f\left(t_{2}\right)= \begin{cases}\alpha \mathrm{e}^{-\alpha\left(t_{2}-T_{2}\right)}, & t_{2} \geq \tau_{2} \\ 0, & t_{2}<\tau_{2}\end{cases}
$$

where $\alpha$ and $\beta$ are called the time constants and $\beta>\alpha>0$, then the form of the probability density function of Equation (2) can be derived from consulting a table of the Laplace transform:

$$
f(t)=\frac{\alpha \beta}{\beta-\alpha}\left[e^{-\alpha\left(t-t_{0}\right)}-e^{-\beta\left(t-t_{0}\right)}\right]
$$

where $t_{0}=\tau_{1}+\tau_{2}$.

Integrating Equation (5) and using logarithmic expansion, we obtain Equation (6) for small $t$ and Equation (7) for large $t$ :

$$
\begin{gathered}
\log [1-F(t)] \simeq \frac{\alpha}{\beta}\left[1-e^{-(\beta-\alpha)\left(t-t_{0}\right)}\right]-\alpha\left(t-t_{0}\right) \\
\log [1-F(t)] \simeq-\alpha\left(t-t_{0}-\frac{1}{\beta}\right)
\end{gathered}
$$

For the fuller details of the derivation of these equations, see McGill (1963, pp. 326-327).

The shortest of the search times obtained was adopted as an estimate of the threshold parameter $t_{0}$. After having estimated $t_{0}$, the estimation of the time constants, $\alpha$ and $\beta$, followed the procedure suggested by McGill (1963, p. 327). The curve shown in Fig. 1 of one target search is the theoretical one predicted by substituting $\hat{\mathrm{t}}_{0}=0.8, \hat{\alpha}=0.118$, and $\hat{\beta}$ $=0.417$ into Equations (6) and (7). On the whole, the fit is satisfactory.

Parallel Processing Model. This type of model is concerned with time distributions for two and three target search. We now consider the general case, i.e., n-target search. As is clear from the above-mentioned conceptual models, in this situation we have in effect $n$ parallel processes to each search response. Therefore, the time consumed in search for the first target number will be the shortest of the $n$ search times. If we suppose that $n$ processes are independent and that they follow the same density function with respect to time, then the probability density function of the shortest time is given by

$$
\mathbf{f}_{\eta}(t)=\eta \mathbf{f}(t)[1-F(t)]^{\eta-1}
$$

We want to use Equation (5) as a form of the density function $f(t)$ in Equation (8). In the present case, however, this raises a mathematical complexity, especially for three target search. As a first-order approximation to Equation (5), therefore, we now assume the following:

$$
f(t)= \begin{cases}\lambda e^{-\lambda\left(t-t_{0}\right)}, & t \geq t_{0} \\ 0 & , \quad t<t_{0}\end{cases}
$$

where $\lambda>0$ and $t_{0}$ is a fixed constant. We obtain immediately that the density function expressed in Equation (8) is

$$
f_{\eta}(t)= \begin{cases}\eta \lambda e^{-\eta \lambda\left(t-t_{0}\right)}, & t \geq t_{0} \\ 0 & , t<t_{0}\end{cases}
$$


where $n=2$ for two target search and $n=3$ for three target search. Integrating Equation (10), we obtain

$$
\log [1-F(t)]=-\eta \lambda\left(t-t_{0}\right)
$$

The straight lines fitted to the data of two and three target searches in Fig. 1 are the theoretical ones predicted from Equation (11). As in the serial processing model, an estimate of the parameter $t_{0}$ was the minimum search time, and $\hat{t}_{0}=0.8$ in both searches. The slope of Equation (11) was estimated by the method of least squares based solely on the data for which linearity holds. Consequently, we get $\hat{\lambda}=0.060$ for two target search and $\hat{\lambda}=0.053$ for three target search. The fits are good at the early stage of search, but they are not so good at the later stage, especially in the case of two target search.

\section{DISCUSSION}

As stated in the introduction, recent studies have been carried out by employing various measures which are available for the purpose of determining whether a human subject processes sensory messages in serial or parallel modes. At the present stage of the investigation, however, results are not always unambiguous. For example, there are several studies which suggest the possibility that the mode of information processing by the $S$ is intrinsically parallel on the basis of the theoretical bias (Neisser, 1963a, b; Neisser et al, 1963). Some investigators, on the other hand, insist that the information processing is carried out serially rather than in parallel (Estes \& Wessel, 1966; McGill, 1963; Stermberg, 1967; Ueno \& Nakaseka, 1967). From these competing views, therefore, it seems most plausible to infer that the $S$ can use both serial and parallel processing modes, and that he utilizes either of them predominantly under a specific condition.

A rigorous approach to the problem of serial vs parallel processings would be to examine the extent to which the observed data may be accounted for by the mathematical models in which the two modes are described. If, in this case, some of the parameters in a model may be specified by any statement about the physical features of the situation to which the model is being applied, then it goes without saying that the model increases its unique representation.
As a result, we will be able to give a solution to the determination of mode of information processing. The design of the present work was based on such methodological considerations.

It is apparent from Fig. 1 that McGill's two-step serial processing model fits well the data of one target search. Equation (6) gives a reasonable description of the early curvature in a latency distribution. The parallel processing model expressed by Equation (11) holds approximately for the searches of two and three targets. In these cases, however, we observe that this model does not satisfactorily handle the long times obtained. This is particularly remarkable in two target search. It seems that the discrepancies between the observed and theoretical values are due either to the breakdown of the independence of parallel searching processes or to the existence of hidden subresponses. Therefore, a more refined model must have a built-in mechanism for generating the curvature at the later stage of search time.

\section{References}

Egeth, H. E. Parallel versus serial processes in multidimensional stimulus discrimination. Percept. \& Psychophys., 1966, 1, 245252.

Estes, W. K., \& Wessel, D. L. Reaction time in relation to display size and correctness of response in forced-choice visual detection. Percept. \& Psychophys., 1966, 1, 369-373.

Fried, C., Rosenfeld, A., \& Gerstman, L. J. Sequential and parallel processing in boundary selections on one-dimensional pictorial stimuli. Percept. mot. Skills, 1965, 20, 789-801.

McGill, W. J. Stochastic latency mechanisms. In R. D. Luce, R. R. Bush, \& E. Galanter (Eds.), Handbook of mathematical psychology. Vol. 1. New York: Wiley. Pp, 309-360.

Neisser, U. Decision-time without reaction-time: Experiments in visual scanning. Amer. J. Psychol., 1963a, 76, 376-385.

Neisser, U. The multiplicity of thought. Brit. J. Psychol., 1963b, 54, 1-14.

Neisser, U., Novick, R., \& Lazar, R. Searching for ten targets simultaneously. Percept. mot. Skills, 1963, 17, 955-961.

Parzen, E. Stochastic processes. San Francisco: Holden-Day, 1962.

Sternberg, S. Two operations in character recognition: Some evidence from reaction-time measurements. Percept. \& Psychophys., $1967,2,45-53$

Ueno, T., \& Nakaseko, M. A serial processing model for tactile scanning. Jap. Psychol. Res., 1967, 9, 62-69.

Weisstein, N. Backward masking and models of perceptual processing. J. exp. Psychol, 1966, 72, 232-240.

(Accepted for publication October 30, 1967.) 